\title{
Electrical Breakdown Mechanisms in Vacuum Diodes
}

\author{
P. Osmokrović ${ }^{a}$, D. Ilić ${ }^{b}$, K. StankoviĆ ${ }^{a}$, M. VujisiĆ ${ }^{a}$ And B. LonČAR ${ }^{c, *}$ \\ ${ }^{a}$ Faculty of Electrical Engineering, University of Belgrade \\ Bulevar Kralja Aleksandra 73, P.O. Box 3554, 11000 Belgrade, Serbia \\ ${ }^{b}$ Mektronik Zemun, 11080 Zemun, Serbia \\ ${ }^{c}$ Faculty of Technology and Metallurgy, University of Belgrade \\ Karnegijeva 4, 11120 Belgrade, Serbia
}

(Received May 4, 2010)

\begin{abstract}
This paper investigates the roles of different mechanisms constituting the process of electrical breakdown in vacuum. Random variable "vacuum breakdown voltage" was measured for pulse breakdowns in five types of vacuum diodes, with different values of vacuum pressure and inter-electrode gap. Conclusions about the influence of vacuum pressure and inter-electrode distance on the parameters of theoretical statistical distributions have been drawn. Based on the distribution to which the "vacuum breakdown voltage" random variable adheres, the dominance of certain breakdown mechanisms has been established for specific ranges of vacuum pressure. The observed absence of anode vacuum breakdown at small inter-electrode gaps has been given a theoretical interpretation.
\end{abstract}

PACS numbers: $51.50+\mathrm{v}$

\section{Introduction}

The goal of this paper is to determine a statistical model which provides a satisfactory description of the "vacuum breakdown voltage" random variable and its connection to the vacuum breakdown mechanism. For that purpose, theoretical and experimental investigations of pulse breakdown voltage statistical properties in two-electrode vacuum-insulated diodes were conducted.

Vacuum breakdown originates in metal pores of the electrode material, i.e., breakdown is initiated by formation of a cloud of evaporated electrode material in which breakdown then develops through the classical gas avalanche mechanism. The crucial point for this process is the creation of a sufficient starting amount of metal pores, and for this purpose it is necessary to provide at least one of the electrodes with enough energy to cause its thermal instability. Thermal instability of one of the electrodes can be caused by the emission mechanism, by accelerated electrode material microparticles or through the avalanche effect in the adsorbed residual gas layer on the electrodes $[1,2]$.

The basis of all emission hypotheses is the electron emission current, caused by a strong electric field and leading to thermal instability of one or both electrodes, whereupon vapour of electrode material is emitted into the inter-electrode region. Gas breakdown further occurs

* corresponding author; e-mail: bloncar@tmf.bg.ac.rs in the evaporated electrode material and desorbed gas. Depending on the electrode at which thermal instability first develops, emission mechanism can be cathode- or anode-initiated [3].

During cathode vacuum breakdown, thermal instability of the cathode happens when the emission current from microspikes exceeds a certain critical value at which the microspikes melt and evaporate due to the Joule effect. The Joule losses produce an irreversible temperature rise, amplifying the electron emission current and additionally increasing resistance of microspikes. This in term causes further rise of the Joule losses and leads to microexplosions of microbulge peaks. Plasma formed in this way is dense enough to afforce electric field at the electrode surface through the Debye field. The forming of cathode plasma follows, which does not spread significantly from the cathode, while the cloud of anode plasma spans across the inter-electrode gap, finally producing the breakdown. On the macroscopic scale, initiation time for cathode vacuum breakdown is under $0.6 \mu \mathrm{s}[4,5]$.

During the anode vacuum breakdown thermal instability of the anode is caused by the energy imparted to it by the electron beam emitted from the cathode, which leads to local heating. This causes anode stain material to melt and evaporate, while at the same time cathode microspikes, the emitters, are thermally stable. This instability develops further, until sufficient density of anode material vapour is established so that the Townsend condition for gas breakdown is met. On the macroscopic scale the time of anode vacuum breakdown initiation is 
longer than $10 \mu \mathrm{s}[6,7]$.

The microparticle breakdown is caused by microparticles, generally present on the electrodes, loosely bound to their surface or free. When voltage is applied to the electrodes these microparticles electrify through electrostatic induction and then accelerate in the inter-electrodes region by the Coulomb force. When microparticles, after multiple charge-exchanging collisions, attain sufficient energy (i.e., speed close to the speed of sound), they evaporate upon collisions. The change of electrode surface topography enhances emission mechanisms off the cathode, which additionally ionize the formed cloud of vapour. Plasma that has thus been formed spreads until the Townsend condition for breakdown is fulfilled in it. On the macroscopic scale microparticle breakdown initiation time is of the order of $10 \mu \mathrm{s}[8,9]$.

The avalanche breakdown hypothesis rests on the assumption of a possible chain process initiation. In collisions with molecules from adsorbed gas layers on the electrodes or with molecules of impurities, multiple charged particles are formed, which move through the inter-electrode region in opposite directions, ionizing new particles on the way, which finally develops into gas breakdown. A prerequisite for such a breakdown to emerge is the presence of adsorbed layers and/or impurities on the electrodes. On the macroscopic scale avalanche breakdown initiation time is of the order of $1 \mu \mathrm{s}$, while the breakdown voltage is expected to be lower than for the other mechanisms of vacuum breakdown. It should be remarked that up to now the hypothesis of avalanche vacuum breakdown has not been undoubtedly confirmed [10].

\section{The experiment}

For determining breakdown voltage values in regions of the $p d$ product in which vacuum breakdowns are expected, vacuum diodes with fixed electrode system were used (Fig. 1). Five kinds of vacuum diodes were manufactured, with different values of pressure and inter-electrode gap, shown in Table. Vacuum pressure was

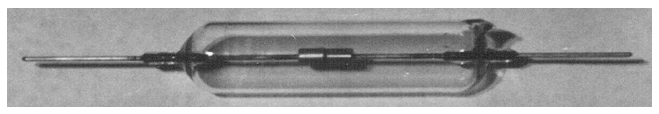

Fig. 1. Vacuum diode with a fixed electrode system.

TABLE

Five vacuum diode types with the corresponding vacuum pressures $p$ and inter-electrode distances $d$.

\begin{tabular}{c|c|c|c|c|c}
\hline \hline $\begin{array}{c}\text { Vacuum } \\
\text { diode type }\end{array}$ & 1 & 2 & 3 & 4 & 5 \\
\hline$p[\mathrm{bar}]$ & $10^{-9}$ & $10^{-9}$ & $10^{-9}$ & $10^{-6}$ & $10^{-4}$ \\
$d[\mathrm{~mm}]$ & 0.1 & 0.3 & 0.5 & 0.1 & 0.1
\end{tabular}

defined by a vacuum system consisting of a mechanical and a diffusion vacuum pump, with the implementation of the degassing process at $480^{\circ} \mathrm{C}$. Tolerance of vacuum pressure set in this way was $\pm 5 \%$. Electrodes were made of electrolytic copper, with surfaces diamond polished to a high gleam. Diode glass container was made of 8245 Jena Schott glass, and current guides of molybdenum wire, which provided a vacuum-tight junction between glass and metal. Inter-electrode gap was determined with $a \pm 10 \mu \mathrm{m}$ tolerance.

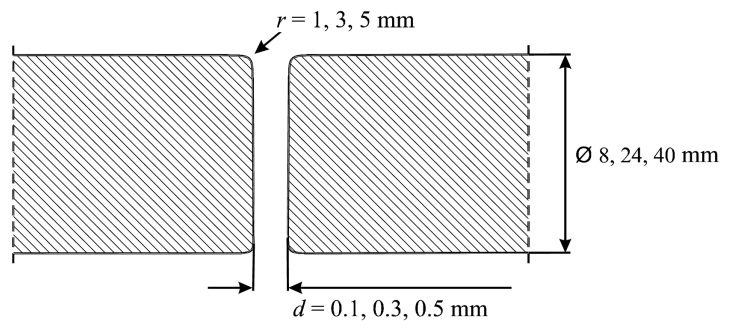

Fig. 2. Profile and dimensions of electrodes.

In Fig. 2 profile and dimensions of vacuum diode electrodes are shown. Electrode edges were rounded with the radius of curvature $r$, to eliminate edge effects. Electrode dimensions and inter-electrode distances were chosen so as to assure homogeneity of the macroscopic electric field. In order to secure the same degree of homogeneity of electric field at different inter-electrode distances, a new pair of electrodes was designed for each distance, whereby, according to the similarity law [11], the following ratio was held: $d: \varnothing=d: r=$ const.

\section{Results and discussion}

For all sets of measured breakdown voltage values, parameters of three statistical distributions were estimated: Weibull, double exponential, and normal. In order to determine which of the three distributions best described the measured values, experimental results were tested for belonging to one of these distributions. Relative values of $\chi^{2}$ test-variable, scaled by the allowed value $\chi_{\text {allowed }}^{2}$ corresponding to $5 \%$ significance level, for the five types of vacuum diodes are presented in Fig. 3 . It can be seen that in high-vacuum conditions $\left(p=10^{-9}\right.$ bar, type 1,2 and 3 vacuum diodes) Weibull distribution is the one best describing the obtained results. In Fig. 4 values of the pulse breakdown voltage at $p=10^{-9}$ bar and $d=0.1 \mathrm{~mm}$ are presented on the probability plotting paper for Weibull distribution.

Values of breakdown voltages for type 4 vacuum diode $\left(d=0.1 \mathrm{~mm}, p=10^{-6}\right.$ bar) are shown in Fig. 5 . It can be seen from this figure that the majority of breakdown voltage values follow the Weibull distribution, which suggests a single breakdown mechanism. However, high-probability breakdown voltages exhibit a clear departure from the Weibull distribution, which can be explained by the appearance of another breakdown mechanism. 


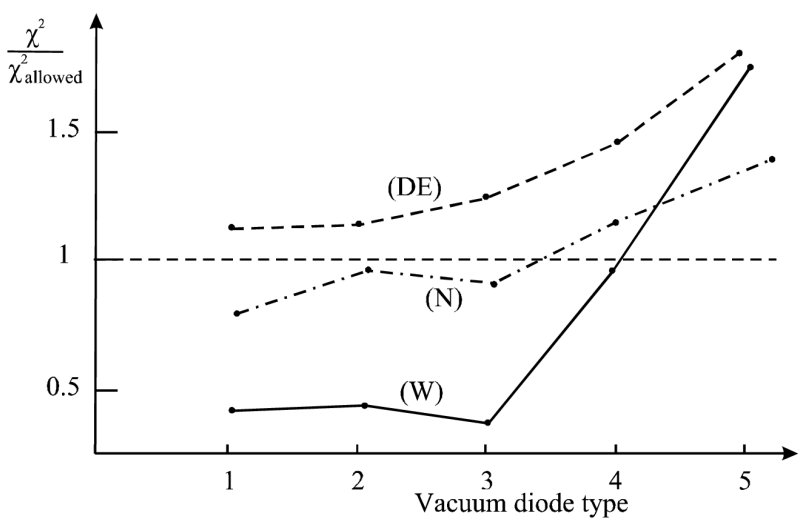

Fig. 3. Relative values of $\chi^{2}$ test-variable scaled by the allowed value $\chi_{\text {allowed }}^{2}$ corresponding to $5 \%$ significance level, for the five types of vacuum diodes. Diode labeling according to Table. (W) - Weibull distribution, (N) - normal distribution, (DE) - double exponential distribution.

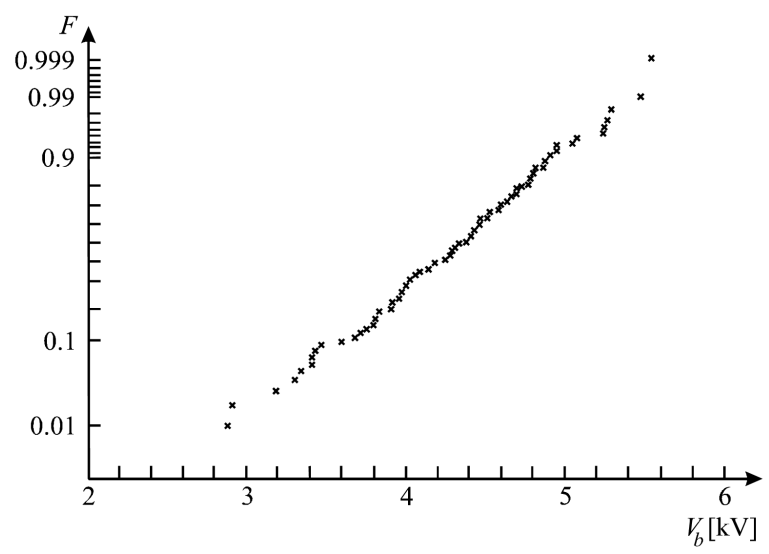

Fig. 4. Vacuum diode pulse breakdown voltage at $p=$ $10^{-9}$ bar and $d=0.1 \mathrm{~mm}$ (plotted on the probability paper for the Weibull distribution).

At $10^{-4}$ bar pressure, this effect is even more pronounced. Figure 6 shows the results for pulse breakdown voltages in type 5 vacuum diode $(d=0.1 \mathrm{~mm}$, $p=10^{-4}$ bar). It is obvious that in this case breakdown voltage values follow a combined distribution, with two parts of it corresponding to two different breakdown mechanisms. Beside the breakdown induced by cathode electron emission, it is most likely that the avalanche mechanism of vacuum breakdown is at work as well, and that the gas multiplicative mechanism is not active. The gas (multiplicative) breakdown mechanism has a low probability of occurring, since the electron mean free path is much larger than the inter-electrode gap. The increase of the avalanche mechanism contribution to breakdown initiation with the rise of pressure can be attributed to the fact that high pressure enlarges the adsorbed gas layer on the electrodes, which leads to a higher probability of avalanche exchange of charge between the elec- trodes. Avalanche vacuum breakdown mechanism was for a long time only a theoretical assumption, without experimental evidence [12]. Results presented in this paper and their interpretation present a justification of the avalanche mechanism concept.

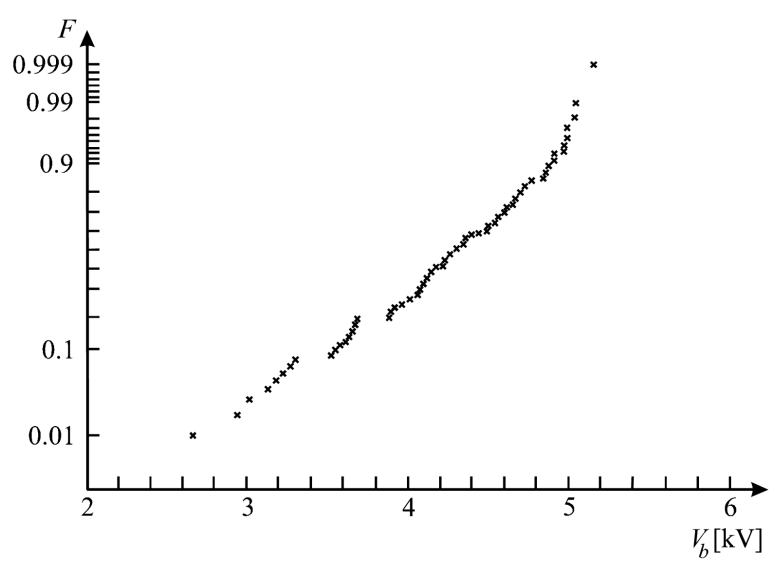

Fig. 5. Vacuum diode pulse breakdown voltage at $p=$ $10^{-6}$ bar and $d=0.1 \mathrm{~mm}$ (plotted on the probability paper for the Weibull distribution).

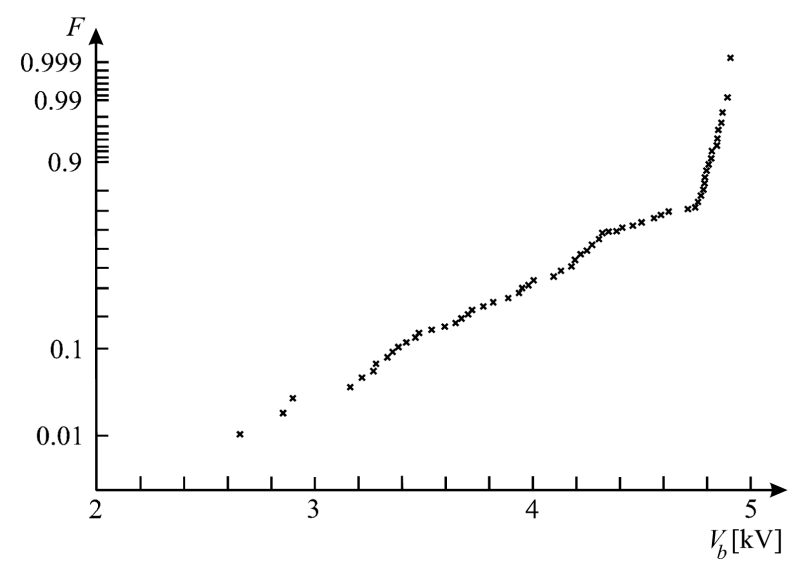

Fig. 6. Vacuum diode pulse breakdown voltage at $p=$ $10^{-4}$ bar and $d=0.1 \mathrm{~mm}$ (plotted on the probability paper for the Weibull distribution).

If instead of the breakdown voltage, critical macroscopic electric field $E_{\mathrm{c}}=V_{\mathrm{b}} / d$ had been investigated as a random variable at a fixed value of pressure, it would have been described by a single Weibull distribution function. The unique distribution function of the critical electric field (which changes negligibly from one series of measurements to another) indicates that cathode emission mechanism is the one generating the breakdown [3]. This conclusion was additionally validated for type 1 vacuum diodes $\left(p=10^{-9}\right.$ bar, $\left.d=0.1 \mathrm{~mm}\right)$ through measurements with a pulse voltage of inverted (positive) polarity. For the new polarity of the applied voltage it was found that diode electrode system was completely de- 
conditioned by previous measurements. However, after conditioning this system with 100 breakdowns, measured values of pulse breakdown voltage were approximately equal to those for negative polarity. The established occurrence of electrode system deconditioning at the change of voltage polarity proves that only cathode is conditioned during breakdown, substantiating the conclusion that breakdown mechanism is of cathode type.

The absence of the anode breakdown mechanism, as well as of the microparticle mechanism, is a consequence of the small inter-electrode distance. A crucial condition for the anode breakdown to occur is that the energy imparted to the anode by the electron beam is sufficient to cause its thermal instability. Since the energy of electrons emitted from the cathode, which collide with the anode, is proportional to the distance between these two electrodes, for small inter-electrode gaps it is insufficient for initiating the anode breakdown mechanism. For the same reason the microparticle breakdown mechanism does not arise. Namely, for a microparticle to evaporate during a collision with an electrode, its velocity needs to be approximately equal to the speed of sound in the material it is made of $(\approx 5 \mathrm{~km} / \mathrm{s})$. In the case of small inter-electrode gaps this condition is hard to achieve. Besides, in experiments with electrode surfaces polished to a high gleam, the possibility of microparticles being present at electrode surfaces is greatly reduced.

\section{Conclusion}

Based on the measurements of pulse vacuum breakdown voltage values, it may be concluded that "vacuum breakdown voltage" random variable is strongly influenced by vacuum pressure. Distribution of the "vacuum breakdown voltage" random variable is best described by the Weibull distribution. At small inter-electrode gap widths, a unique distribution is valid when critical macroscopic electric field is taken for a random variable. At $10^{-4}$ bar pressure and for the inter-electrode gap of $0.1 \mathrm{~mm}$ breakdown occurs through a combination of two vacuum mechanisms: cathode and avalanche.
At $10^{-6}$ bar pressure and for the inter-electrode gap of $0.1 \mathrm{~mm}$, breakdown still occurs through the combination of cathode and avalanche mechanisms, with a considerably reduced contribution of the avalanche mechanism compared to the $10^{-4}$ bar pressure, $0.1 \mathrm{~mm}$ inter-electrode gap case. The decrease of the avalanche mechanism contribution can be attributed to the decrease of the adsorbed gas layer on the electrodes when pressure is lowered. At $10^{-9}$ bar pressure and for inter-electrode gaps of $0.1 \mathrm{~mm}, 0.3 \mathrm{~mm}$ and $0.5 \mathrm{~mm}$, breakdown occurs entirely through the cathode mechanism.

\section{Acknowledgments}

The Ministry for Science and Technological Development of the Republic of Serbia supported this work under contract 141046 .

\section{References}

[1] Electrical Breakdown of Gases, Eds. J.M. Meek, J.D. Crags, Wiley, New York 1978.

[2] B. Loncar, P. Osmokrovic, A. Vasic, S. Stankovic, IEEE Trans. Plasma Sci. 34, 1561 (2006).

[3] P. Osmokrovic, N. Kartalovic, IEEE Trans. Power Syst. 12, 1455 (1997).

[4] G.E. Vibrans, J. Appl. Phys. 35, 2855 (1964).

[5] P.A. Chatterton, Proc. Phys. Soc. Lond. 88, 231 (1966).

[6] D.W. Williams, W.T. Williams, J. Phys. D, Appl. Phys. 5, 280 (1972).

[7] D.K. Davies, M.A. Biondi, J. Appl. Phys. 42, 3089 (1971).

[8] P.A. Chatterton, M.M. Menon, K.D. Srivastava, J. Appl. Phys. 43, 4536 (1972).

[9] R.V. Latham, J. Phys. D, Appl. Phys. 5, 2049 (1972).

[10] P.A. Chatterton, in Ref. [1], p. 75.

[11] P. Osmokrović, T. Živić, B. Lončar, A. Vasić, Plasma Sources Sci. Technol. 15, 703 (2006).

[12] G.R. Werner, Ph.D. Thesis, Cornell Univ., 2004. 